\title{
High Grade Prostatic Intraepithelial Neoplasia, Mucinous Variant
}

National Cancer Institute

\section{Source}

National Cancer Institute. High Grade Prostatic Intraepithelial Neoplasia, Mucinous

Variant. NCI Thesaurus. Code C39888.

High grade prostatic intraepithelial neoplasia characterized by the presence of masses of mucin within the affected distended glands. 